\section{§ 20. Electron Temperature Gradient Instability in Toroidal Plasmas}

Dong, J.Q. (SWIP, China)

Sanuki, H., Itoh, K.

Chen Liu (UC Irvine, USA)

The cross-field particle, momentum, and energy transport in magnetically confined fusion plasmas generally exceeds neoclassical predictions by more than one order of magnitude. There is strong experimental evidence that the anomaly can be attributed to a variety of smallscale turbulence driven by gradients of plasma parameters such as density, velocity and temperature. Especially, the temperature gradient (TG) driven instabilities were proposed as the plausible candidates responsible for the anomalous thermal transport. The linear and nonlinear Ion temperature gradient (ITG) instability theories and simulations are enough to explain the ion transport anomaly well. However, the electron thermal transport is often still anomalous even in discharges with an ITB. Recent experimental observations support the hypothesis that, within an ITB, ITG turbulence is suppressed by the shear flow, whereas the electron temperature gradient (ETG) driven turbulence may control the electron thermal transport. So, ETG instability and anomalous electron transport becomes a hot topic in recent years.

In this study, ETG driven instability in toroidal plasmas is investigated with gyrokinetic theory. The full electron kinetics including fineite Larmor radius effects, toroidal drift motion and transit $k_{/ /} v_{/ /}$is considered. The upgraded numerical scheme for solving the integral eigenvalue equation allows the study of both growing and damping modes and thus direct calculation of critical gradient. A systematic parameter study is carried out for low beta circular flux equilibrium. The scaling of the critical gradient with respect to toroidicity is obtained. We solve the coupled integral dispersion equations numerically and studied eigen mode structure in both configuration and $\mathrm{k}$ spaces. Since the recent experimental data indicate clearly that the normalized electron thermal diffusivity $\chi_{e} / T_{e}^{1.5}$ vs. $\left(\nabla T_{e} / T_{e}\right), \quad$ exhibits universal behavior for plasmas. Finally we obtained the following scaling for the electron thermal diffusivity,

$$
\begin{aligned}
& \chi_{e} \approx \frac{D_{B}}{0.0173 \tau_{i}^{2}+1.95 \tau_{i}+1.17} \\
& \times \frac{c}{\omega_{p e}}\left[\frac{1}{L_{T e}}-\left(\frac{1}{L_{T e}}\right)^{c}\right] F\left(\varepsilon_{n}, \hat{s}, q\right)
\end{aligned}
$$

where $D_{B}=c T_{e} / e B$ is the diffusion coefficient by Bohm. This scaling is in qualitatively agreement with the experimental observations.

1) J. Q. Dong, H. Sanuki, K. Itoh and Liu Cheng ; IAEA, TH/1-6.

2) Physics Plasmas, 9, 4699(2002). 